\title{
Quantization of relativistic fields without zero-point energy 1. A complex scalar field
}

\begin{abstract}
Zahid Zakir *
Abstract

The relativistic fields having opposite sign on frequency modes usually are represented as complex fields only with positive-frequency modes, but with global $\mathrm{U}(1)$ symmetry and a charge conjugation (C-symmetry). However, at conventional introduction of operators of antiparticles C-symmetry becomes broken and then a zero-point charge and a vacuum zero-point energy arises. The new method of quantization of relativistic fields without C-symmetry violation is developed. On an example of a complex scalar field it is shown that $\mathrm{C}$-symmetry requirements impose such constraints on bilinear products of ladder operators which lead to new operator identities. They then allow to express the observables through charge-conjugate creation-annihilation operators at once in a normal-ordered form without the zeropoint energy and the zero-point charge. It is shown that a self-interaction of a complex scalar field does not change the vacuum energy. As the invariant propagators of particles and antiparticles can be chosen the retarded and advanced propagators accordingly.
\end{abstract}

PACS: 03.70.+k; 11.10.-z; 11.30.Er

Key words: vacuum energy, vacuum fluctuations, time reversal, charge conjugation

\section{Content}

Introduction

1. Canonical formalism for a complex scalar field

24

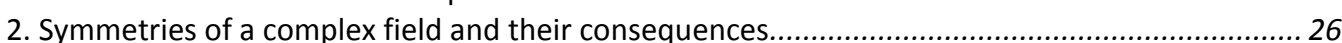

3. The vacuum energy with the self-energy. First order............................................................. 28

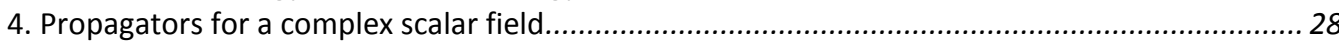

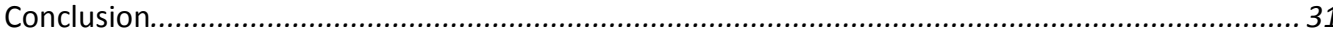

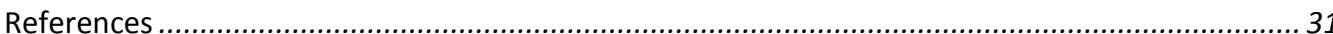

\section{Introduction}

In the previous papers $[1,2]$ it has been shown that standard quantization rules of the positive-frequency nonrelativistic harmonic oscillator have not been adequately adapted for quantization of the systems having both positive- and negativefrequency modes. In the such systems initially there is a symmetry under interchanging of quanta of opposite sign of frequency, at which changes a sign of their Hamiltonians. After the interpretation of negative-frequency quanta as positive-frequency antiquanta with opposite sign of a charge, this symmetry transforms to the charge conjugation symmetry ( $C$-symmetry) at which changes a sign of their charges only.

However, at usual procedures of quantization of relativistic fields there were broken both forms of this discrete symmetry. The first by a divergent zero-point

"Centre for Theoretical Physics and Astrophyics, Tashkent, Uzbekistan; zahidzakir@theor-phys.org 
energy, and the second by a divergent zero-point charge. Then, these symmetries formally became restored after the erasing of these divergent constants.

In the papers [1] a natural modification of the standard procedures of quantization has been proposed where instead of artificial hypotheses and procedures only the restrictions following from $C$-symmetry have been used. As the result, for the $C$ symmetric systems the zero-point energy and the zero-point charge disappear.

At disputes about this formalism four objections mainly have been discussed.

The first one (with which all opponents began) is that the existence of the zero-point fluctuations of vacuum is experimentally proved by several effects and that this fact is conventional. The answer to this objection is that the Lamb shift and the Cazimir effect, to which they refer at this case, are exactly described by interactions of real sources without a zero-point energy of vacuum and this fact also is wellknown [1]. Moreover, most of consecutive thinking authors noted that, if the zeropoint energy would really exists, it would lead to a catastrophic inconsistency between particle physics and cosmology. Thus, the experiments testify about lack, instead of existence, of the zero-point fluctuations of vacuum, so the problem is how to coincide this fact with principles of quantum field theory.

The second objection was about the using of negative-frequency states which would have a negative norm. This objection disappears if initially we introduce operators of antiparticles by a way, as it is accepted in theories of relativistic fields. In the previous paper about a complex harmonic oscillator [2] and in the present paper the such version of a new method is considered. A more fundamental solution of the negative norm problem is presented in [3].

The third objection concerned renormalization problems that even if to exclude a constant in the unperturbed Hamiltonian, the interactions will give a divergent contribution to the vacuum energy, especially, if to take into account quantum effects of gravitation. As shown in [2] and it will be considered in the present paper also, most promptly growing contributions to the vacuum energy - the zero-point fluctuations and first order contributions - disappear thanks to $C$-symmetry requirements.

Notice, that instead of a causal propagator, the discrete symmetries formally allow us to use the retired and advanced propagators, disappearing beyond the light cone and less singular at zero, which physically more attractive.

At last, any controversy has been terminated by the last argument, that though there are no special objections against a new method as a formalism, but the zero-point fluctuations of fields should be real at least because of the uncertainty relations and that this is a strong requirement of quantum mechanics. This is fourth objection after which the new method has been treated as a mathematical trick not having a physical meaning.

The answer to this last objection, given in the previous paper [2], is that the Heisenberg uncertainty relation $\delta x^{2} \delta p^{2} \geq \hbar^{2} / 4$ have been derived for systems with real valued canonical variables $x, p$. If to quantize systems with non-Hermitian (complex) canonical variables $q, q^{*}$ and $p, p^{*}$, which concern the most of relativistic fields, there is more general form of uncertainty relations: 


$$
\delta|q|^{2} \delta|p|^{2} \geq \frac{\hbar^{2}}{4}\langle Q\rangle^{2},
$$

where $\langle Q\rangle=i\left\langle n\left|q^{*} p^{*}-p q\right| n\right\rangle / \hbar$ is a charge of the state fluctuations of which are considered.

For hermitian variables the expression in brackets in $\langle Q\rangle$ turns to the standard commutator $i(q p-p q) / \hbar=-1$ and the relation (1) transfers to the standard uncertainty relation. Then, of course, the zero-point energy for a harmonic oscillator ground state is inevitable.

However, for the systems with complex canonical variables and a chargeconjugation symmetry, at their ground state $\langle Q\rangle=0$ and from (1) follows, that $\delta|q|^{2} \delta|p|^{2} \geq 0$. It means that the ground state of the such system is not quantized and does not fluctuate. Then the (unperturbed) vacuum of relativistic fields is analogous to an external field, the energy density of which does not fluctuate and is defined by a classical Hamiltonian.

In the present paper the new method is applied to quantization of a complex relativistic scalar field. In the first section the canonical formalism for the field is presented. Thus, it appears that the operators $\beta_{k}^{*}, \beta_{k}$, introduced at the frequency decomposition of fields and usually interpreted as operators of antiparticles, are not charge-conjugate to operators of particles. Therefore, in section 2 the $\mathrm{C}$-symmetry requirements are considered, allowing to introduce operators of antiparticles $b_{k}^{*}, b_{k}$ as charge-conjugate ones to the operators of particles and to obtain an operator identity

$$
b_{k}^{*} b_{k}=\beta_{k} \beta_{k}^{*} \text {. }
$$

This identity then leads to vanishing of a zero-point charge and a zero-point energy of vacuum and first order contributions also (section 3). In section 4 the invariant propagators of the field are discussed.

\section{Canonical formalism for a complex scalar field}

In the theory of relativistic fields positive- and negative-frequency states appear in a symmetric form and, as a result, the number of degrees of freedom of a relativistic field appears doubled in comparison with the same nonrelativistic field. One of ways of the account of this doubling of degrees of freedom is the representation of the relativistic field as a complex field.

Lagrangian of a complex scalar field $\phi=\phi_{1}+i \phi_{2}$ :

$$
L=\int d^{3} x\left[\left(\partial_{\mu} \phi^{*}\right)\left(\partial^{\mu} \phi\right)-m^{2} \phi^{*} \phi\right],
$$

gives the canonical momenta $\pi(x)=\partial_{t} \phi^{*}, \pi^{*}(x)=\partial_{t} \phi$ and the Hamiltonian:

$$
H=\int d^{3} x\left(\pi \pi^{*}+m^{2} \phi^{*} \phi\right) .
$$

Notice, that at including of interaction with gauge fields different orderings lead to different charge operators. Here we will use the standard ordering as in (3)-(4). 
The field equations for the complex field:

$$
\partial_{\mu} \partial^{\mu} \phi-m^{2} \phi=0, \quad \partial_{\mu} \partial^{\mu} \phi^{*}-m^{2} \phi^{*}=0
$$

have the general solutions with both positive- and negative-frequency modes:

$$
\begin{aligned}
& \phi(x)=\sum_{\mathbf{k}}\left(a_{k} e^{-i k x}+a_{-k} e^{i k x}\right), \quad \sum_{\mathbf{k}} \equiv \int \frac{d^{3} \mathbf{k}}{\sqrt{(2 \pi)^{3} 2 \omega_{\mathbf{k}}}} . \\
& \phi^{*}(x)=\sum_{\mathbf{k}}\left(a_{k}^{*} e^{i k x}+a_{-k}^{*} e^{-i k x}\right) .
\end{aligned}
$$

At quantization the equal time commutators should be introduced:

$$
i\left[\pi(\mathbf{x}, t), \phi\left(\mathbf{x}^{\prime}, t\right)\right]=\delta^{3}\left(\mathbf{x}-\mathbf{x}^{\prime}\right), \quad i\left[\pi^{*}(\mathbf{x}, t), \phi^{*}\left(\mathbf{x}^{\prime}, t\right)\right]=\delta^{3}\left(\mathbf{x}-\mathbf{x}^{\prime}\right) .
$$

In the present paper we exclude the negative-frequency operators, as it is accepted in the standard quantum theory of relativistic fields, by introducing a positive-frequency operator $\beta_{k}^{*}$ instead of an annihilation operator $a_{-k}$ of negativefrequency quanta. As a result, two forms of quanta with opposite signs of frequency will be presented as two forms of quanta with opposite charges.

Then, the frequency decomposition of fields takes the standard form:

$$
\begin{array}{ll}
\phi(x)=\sum_{\mathbf{k}}\left(a_{k} e^{-i k x}+\beta_{k}^{*} e^{i k x}\right), & \pi^{*}(x)=-i \sum_{\mathbf{k}} \omega_{\mathbf{k}}\left(a_{k} e^{-i k x}-\beta_{k}^{*} e^{i k x}\right), \\
\phi^{*}(x)=\sum_{\mathbf{k}}\left(a_{k}^{*} e^{i k x}+\beta_{k} e^{-i k x}\right), & \pi(x)=i \sum_{\mathbf{k}} \omega_{\mathbf{k}}\left(a_{k}^{*} e^{i k x}-\beta_{k} e^{-i k x}\right) .
\end{array}
$$

Since the field operators $\phi^{*}, \phi$ and $\pi^{*}, \pi$ are non-Hermitian, two sets of creationannihilation operators $a_{k}^{*}, a_{k}$ and $\beta_{k}^{*}, \beta_{k}$ describe different kind of particles and they commutate with each other. Corresponding non-zero commutators for these operators, following from (8), are:

$$
\left[a_{k}, a_{k^{\prime}}^{*}\right]=\delta^{3}\left(\mathbf{k}-\mathbf{k}^{\prime}\right), \quad\left[\beta_{k}, \beta_{k^{\prime}}^{*}\right]=\delta^{3}\left(\mathbf{k}-\mathbf{k}^{\prime}\right) .
$$

The Hamiltonian (4) then takes a form:

$$
H=\int d^{3} k H_{k}=\int d^{3} k \omega_{k}\left(a_{k}^{*} a_{k}+\beta_{k} \beta_{k}^{*}\right) .
$$

Here the operator product $\beta_{k} \beta_{k}^{*}$ has not a normal-ordered form and it was main difficulty of former treatments. Thus, the operators $\beta_{k}^{*}, \beta_{k}$ were assumed to be charge-conjugate to operators of particles $a_{k}^{*}, a_{k}$. As a result of this intuitive hypothesis, then, after the replacement $\beta_{k} \beta_{k}^{*}=\beta_{k}^{*} \beta_{k}+1$, there arisen a zero-point energy and a zero-point charge of the vacuum.

However, as it will be shown further in the next section, the such interpretation of the operators appears incorrect, since then the $C$-symmetry requirements become violated. In fact, there are other operators $b_{k}^{*}, b_{k}$ obtained by the charge conjugation of the operators of particles $a_{k}^{*}, a_{k}$, and they, because of $C$ symmetry requirements, are related with the operators $\beta_{k}^{*}, \beta_{k}$ non-linearly through the considered relation (2). 


\section{Symmetries of a complex field and their consequences}

In the system described by a complex field with a Lagrangian (3) there is a global $U(1)$ symmetry:

$$
\phi^{\prime}(x)=U \phi(x) U^{-1}, \quad U=\exp (i \theta Q)
$$

with conserved charge $\partial_{t} Q=0$. Here $Q$ is the charge operator defined as:

$$
Q=i \int d^{3} x\left[\phi^{*} \partial_{t} \phi-\left(\partial_{t} \phi^{*}\right) \phi\right]=i \int d^{3} x\left(\phi^{*} \pi^{*}-\pi \phi\right),
$$

which after the frequency decomposition of fields takes a form:

$$
Q=\int d^{3} k Q_{k}=\int d^{3} k\left(a_{k}^{*} a_{k}-\beta_{k} \beta_{k}^{*}\right) .
$$

Notice, that we use the standard ordering and do not consider more complicated forms of ordering.

Since two forms of quanta have the same masses and different charges, there is the symmetry under the charge conjugation $(C)$ transformation at which the Hamiltonian is invariant and the charge changes a sign:

$$
H_{c} \equiv C H C^{-1}=H, \quad Q_{c} \equiv C Q C^{-1}=-Q .
$$

Since the complex field is presented as a set of independent complex oscillators, the $C$-symmetry requirements are valid for each of oscillators separately:

$$
H_{k c} \equiv C H_{k} C^{-1}=H_{k}, \quad Q_{k c} \equiv C Q_{k} C^{-1}=-Q_{k} .
$$

The existence of this symmetry operation leads to the existence of the operators which are charge-conjugate to earlier introduced ones:

$$
\begin{aligned}
& b_{k}=C a_{k} C^{-1}, \quad b_{k}^{*}=C a_{k}^{*} C^{-1}, \\
& \alpha_{k}=C \beta_{k} C^{-1}, \quad \alpha_{k}^{*}=C \beta_{k}^{*} C^{-1},
\end{aligned}
$$

with commutators:

$$
\left[b_{k}, b_{k^{\prime}}^{*}\right]=\delta^{3}\left(\mathbf{k}-\mathbf{k}^{\prime}\right), \quad\left[\alpha_{k}, \alpha_{k^{\prime}}^{*}\right]=\delta^{3}\left(\mathbf{k}-\mathbf{k}^{\prime}\right) .
$$

The creation-annihilation operators $a_{k}, a_{k}^{*}$ and $b_{k}, b_{k}^{*}$ define the vacuum and excited states $\left|n_{ \pm}\right\rangle$containing mutually charge-conjugate quanta (particles and antiparticles):

$$
\begin{array}{ll}
a_{k}\left|n_{+}\right\rangle=\sqrt{n_{+}}\left|n_{+}-1\right\rangle, & a_{k}^{*}\left|n_{+}\right\rangle=\sqrt{n_{+}+1}\left|n_{+}+1\right\rangle, \\
b_{k}\left|n_{-}\right\rangle=\sqrt{n_{-}}\left|n_{-}-1\right\rangle, & b_{k}^{*}\left|n_{-}\right\rangle=\sqrt{n_{-}+1}\left|n_{-}+1\right\rangle .
\end{array}
$$

At $n_{+}=0, n_{-}=0$ we have $a_{k}\left|0_{+}\right\rangle=0$ and $b_{k}\left|0_{-}\right\rangle=0$.

The corresponding charge-conjugate field operators and momenta:

$$
\begin{array}{ll}
\phi_{c}(x)=\sum_{\mathbf{k}}\left(b_{k} e^{-i k x}+\alpha_{k}^{*} e^{i k x}\right), & \phi_{c}^{*}(x)=\sum_{\mathbf{k}}\left(b_{k}^{*} e^{i k x}+\alpha_{k} e^{-i k x}\right), \\
\pi_{c}(x)=i \sum_{\mathbf{k}} \omega_{\mathbf{k}}\left(b_{k}^{*} e^{i k x}-\alpha_{k} e^{-i k x}\right), & \pi_{c}^{*}(x)=-i \sum_{\mathbf{k}} \omega_{\mathbf{k}}\left(b_{k} e^{-i k x}-\alpha_{k}^{*} e^{i k x}\right) .
\end{array}
$$

satisfy the equal time commutation relations:

$$
i\left[\pi_{c}(\mathbf{x}, t), \phi_{c}\left(\mathbf{x}^{\prime}, t\right)\right]=\delta^{3}\left(\mathbf{x}-\mathbf{x}^{\prime}\right), \quad i\left[\pi_{c}^{*}(\mathbf{x}, t), \phi_{c}^{*}\left(\mathbf{x}^{\prime}, t\right)\right]=\delta^{3}\left(\mathbf{x}-\mathbf{x}^{\prime}\right) .
$$


The charge-conjugate Hamiltonian $H_{c}$ and charge operator $Q_{c}$ are expressed through new operators as:

$$
\begin{aligned}
& H_{c}=\int d^{3} k H_{k c}=\int d^{3} k \omega_{k}\left(b_{k}^{*} b_{k}+\alpha_{k} \alpha_{k}^{*}\right), \\
& Q_{c}=\int d^{3} k Q_{k c}=\int d^{3} k\left(b_{k}^{*} b_{k}-\alpha_{k} \alpha_{k}^{*}\right) .
\end{aligned}
$$

Further, the $C$-symmetry requirements (16) we write in details as:

$$
\begin{aligned}
& H_{k}=\omega_{k}\left(a_{k}^{*} a_{k}+\beta_{k} \beta_{k}^{*}\right)=\omega_{k}\left(b_{k}^{*} b_{k}+\alpha_{k} \alpha_{k}^{*}\right)=H_{k c}, \\
& Q_{k}=\left(a_{k}^{*} a_{k}-\beta_{k} \beta_{k}^{*}\right)=-\left(b_{k}^{*} b_{k}-\alpha_{k} \alpha_{k}^{*}\right)=-Q_{k c},
\end{aligned}
$$

and then obtain two constraint equations for the bilinear operator products:

$$
\begin{aligned}
& a_{k}^{*} a_{k}+\beta_{k} \beta_{k}^{*}=b_{k}^{*} b_{k}+\alpha_{k} \alpha_{k}^{*}, \\
& a_{k}^{*} a_{k}-\beta_{k} \beta_{k}^{*}=-b_{k}^{*} b_{k}+\alpha_{k} \alpha_{k}^{*} .
\end{aligned}
$$

By adding and subtracting these equalities, two products of auxiliary operators $\beta_{k} \beta_{k}^{*}$ and $\alpha_{k} \alpha_{k}^{*}$ we can express through two products of the mutually charge-conjugate operators $a_{k}^{*} a_{k}$ and $b_{k}^{*} b_{k}$ as:

$$
\alpha_{k} \alpha_{k}^{*}=a_{k}^{*} a_{k}, \quad \beta_{k} \beta_{k}^{*}=b_{k}^{*} b_{k} .
$$

These operator identities are those restrictions which put on the system the existence of the $C$-symmetry.

Further, by substituting these relations into the expressions for the Hamiltonian (11) and the charge (14), we obtain the final result:

$$
\begin{aligned}
& H=H_{c}=\int d^{3} k H_{k c}=\int d^{3} k \omega_{k}\left(a_{k} a_{k}^{*}+b_{k}^{*} b_{k}\right), \\
& Q=-Q_{c}=\int d^{3} k\left(a_{k} a_{k}^{*}-b_{k}^{*} b_{k}\right) .
\end{aligned}
$$

Thus, a nontrivial fact is that the creation-annihilation operators of antiparticles $b_{k}^{*}, b_{k}$ are related to the field operators not directly, but through the auxiliary operators $\beta_{k}^{*}, \beta_{k}$ and the bilinear relations (25).

If, as it has been accepted in quantum field theory earlier, we would identify directly the operators $\beta_{k}^{*}, \beta_{k}$ with $b_{k}^{*}, b_{k}$, interpreting $\beta_{k}^{*}, \beta_{k}$ as the creationannihilation operators of antiparticles, we would come to violation of the $C$-symmetry requirements. Really, the conventional intuitive hypothesis

$$
\beta_{k}=b_{k}, \beta_{k}^{*}=b_{k}^{*},
$$

means, according to (25), $\beta_{k} \beta_{k}^{*}=\beta_{k}^{*} \beta_{k}$ and $b_{k} b_{k}^{*}=b_{k}^{*} b_{k}$, which after the charge conjugation lead to $a_{k} a_{k}^{*}=a_{k}^{*} a_{k}$. Since the creation-annihilation operators cannot commutate, hence, the hypothesis (27) and the $C$-symmetry requirements (16) are incompatible. Thus, the usual approach in fact means a failure from the $C$-symmetry requirements.

Contrary to the such approach leading to internal inconsistencies in the theory, we strongly follow the C-symmetry requirements (16) and reject unreasonable 
hypothesis (27). Then, the symmetry requirements (16) allow us to find the nontrivial relations (25) between the auxiliary operators and the true operators of antiparticles.

Thus, the energy and the charge of vacuum of the free relativistic field vanish due to the charge-conjugation symmetry of the system. In the following section we will consider, how the self-interaction of a scalar field contribute to the vacuum energy.

\section{The vacuum energy with the self-energy. First order.}

Symmetries of the complex field admit only gauge-invariant potentials of the form $\left(\phi^{*} \phi\right)^{n}$ and $\left(\phi_{c}^{*} \phi_{c}\right)^{n}$ (including their products), conserving a charge and consequently further we will consider only the such interactions. The standard 4order potential looks like:

$$
V=\lambda\left(\phi^{*} \phi+\phi_{c}^{*} \phi_{c}\right)^{2}
$$

The contribution to the vacuum energy in the first order of perturbation theory is given by the matrix element:

$$
\begin{aligned}
& \left\langle 0\left|V^{(1)}\right| 0\right\rangle=\lambda \int_{-T}^{T} d t \int d^{3} x\left\langle 0\left|\phi^{*}(x) \phi^{*}(x) \phi(x) \phi(x)\right| 0\right\rangle= \\
& =\lambda \int_{-T}^{T} d t \int d^{3} x \sum_{\mathbf{k} k^{\prime} \mathbf{q} q^{\prime}} e^{-i k x-i k^{\prime} x+i q x+i q^{\prime} x}\left\langle 0\left|a_{q^{*}}^{*} a_{k} \beta_{k^{\prime}} \beta_{q}^{*}+\beta_{k^{\prime}} \beta_{q^{\prime}} \beta_{k}^{*} \beta_{q}^{*}\right| 0\right\rangle=0 .
\end{aligned}
$$

This matrix element is described by the diagram of "butterfly" type and, since the field operators commutate at the same spacetime point, the matrix elements from all other orderings of the field operators also disappear.

Thus, in the first order of perturbation theory there are no contributions to the vacuum energy from self-interaction of the complex scalar field.

Notice, that contributions to the vacuum energy of the one-particle states of some "bubble" type diagrams also disappear:

$$
\begin{aligned}
& \langle 1,0|V| 1,0\rangle \sim \lambda \int d^{4} x\left\langle 1,0\left|\left(\phi^{*} \phi\right)^{2}\right| 1,0\right\rangle=0, \\
& \langle 0,1|V| 0,1\rangle \sim \lambda \int d^{4} x\left\langle 0,1\left|\left(\phi_{c}^{*} \phi_{c}\right)^{2}\right| 0,1\right\rangle=0 .
\end{aligned}
$$

The such contributions and loop diagrams will be considered in further papers.

\section{Propagators for a complex scalar field}

The causal propagator for a scalar field in the standard treatment has been defined as a vacuum expectation from the chronological product of fields:

$$
\begin{aligned}
i G_{c}\left(x^{\prime}-x\right) & =\left\langle 0\left|T \phi\left(x^{\prime}\right) \phi^{*}(x)\right| 0\right\rangle= \\
& =\theta\left(t^{\prime}-t\right)\left\langle 0\left|\phi\left(x^{\prime}\right) \phi^{*}(x)\right| 0\right\rangle+\theta\left(t-t^{\prime}\right)\left\langle 0\left|\phi^{*}(x) \phi\left(x^{\prime}\right)\right| 0\right\rangle .
\end{aligned}
$$

In this case in the field operators the hypothesis (27) has been used, which after the momentum decomposition leads to $\left\langle 0\left|\beta_{k^{\prime}} \beta_{k}^{*}\right| 0\right\rangle=\delta^{3}\left(\mathbf{k}^{\prime}-\mathbf{k}\right)$, while other matrix element $\left\langle 0\left|\beta_{k}^{*} \beta_{k}\right| 0\right\rangle=0$ disappears. 
In our case these matrix elements are interchanged the position and, in fact, because of identities (25), we have now for them the opposite values:

$$
\begin{aligned}
& \left\langle 0\left|\beta_{k^{\prime}} \beta_{k}^{*}\right| 0\right\rangle=0, \\
& \left\langle 0\left|\beta_{k^{\prime}}^{*} \beta_{k}\right| 0\right\rangle=\left\langle 0\left|\beta_{k^{\prime}} \beta_{k}^{*}\right| 0\right\rangle-\delta^{3}\left(\mathbf{k}^{\prime}-\mathbf{k}\right)=-\delta^{3}\left(\mathbf{k}^{\prime}-\mathbf{k}\right) .
\end{aligned}
$$

Therefore, it is necessary to construct the propagator of the field which takes into account these modifications.

For this purpose we will consider all four possible two-point functions of a complex field, using momentum decompositions. The vacuum expectations $\left\langle 0\left|\phi(x) \phi\left(x^{\prime}\right)\right| 0\right\rangle$ and $\left\langle 0\left|\phi^{*}(x) \phi^{*}\left(x^{\prime}\right)\right| 0\right\rangle$ obviously vanish, but now the diagonal two-point functions $\left\langle 0\left|\phi^{*}(x) \phi\left(x^{\prime}\right)\right| 0\right\rangle$ and $\left\langle 0\left|\phi_{c}^{*}(x) \phi_{c}\left(x^{\prime}\right)\right| 0\right\rangle$ vanish also:

$$
\begin{aligned}
& \left\langle 0\left|\phi^{*}(x) \phi\left(x^{\prime}\right)\right| 0\right\rangle=\sum_{k k^{\prime}}\left(\left\langle 0\left|a_{k}^{*} a_{k^{\prime}}\right| 0\right\rangle e^{-i k^{\prime} x^{\prime}+i k x}+\left\langle 0\left|\beta_{k} \beta_{k^{\prime}}^{*}\right| 0\right\rangle e^{i k^{\prime} x^{\prime}-i k x}\right)= \\
& =\sum_{k k^{\prime}}\left(\left\langle 0\left|a_{k}^{*} a_{k^{\prime}}\right| 0\right\rangle e^{-i k^{\prime} x^{\prime}+i k x}+\left\langle 0\left|b_{k^{\prime}}^{*}, b_{k}\right| 0\right\rangle e^{i k^{\prime} x^{\prime}-i k x}\right)=0 .
\end{aligned}
$$

As a result, the last vacuum expectation is simply equal to the commutator of the fields and looks like:

$$
\begin{aligned}
& \left\langle 0\left|\phi\left(x^{\prime}\right) \phi^{*}(x)\right| 0\right\rangle=\sum_{k^{\prime} k}\left\langle 0\left|\left(a_{k^{\prime}} e^{-i k^{\prime} x^{\prime}}+\beta_{k^{\prime}}^{*} e^{i k^{\prime} x^{\prime}}\right)\left(a_{k}^{*} e^{i k x}+\beta_{k} e^{-i k x}\right)\right| 0\right\rangle= \\
& =\sum_{k k^{\prime}}\left(\left\langle 0\left|a_{k^{\prime}} a_{k}^{*}\right| 0\right\rangle e^{-i k^{\prime} x^{\prime}+i k x}+\left\langle 0\left|\beta_{k^{\prime}}^{*}, \beta_{k}\right| 0\right\rangle e^{i k^{\prime} x^{\prime}-i k x}\right)= \\
& =\int \frac{d^{3} k}{(2 \pi)^{3} 2 \omega_{k}} e^{i \mathbf{k}\left(\mathbf{x}^{\prime}-\mathbf{x}\right)}\left(e^{-i \omega_{k}\left(t^{\prime}-t\right)}-e^{i \omega_{k}\left(t^{\prime}-t\right)}\right)= \\
& =-i\left[G_{r e t}\left(x^{\prime}-x\right)-G_{a d v}\left(x^{\prime}-x\right)\right]=-i G\left(x^{\prime}-x\right) .
\end{aligned}
$$

and two-point function appears as automatically chronologically ordered. Thus, if $t^{\prime}-t>0$ contributes only the retarding propagator $G_{r e t}\left(x^{\prime}-x\right)$, and at $t^{\prime}-t<0$ only the advanced propagator $G_{a d v}\left(x^{\prime}-x\right)$.

Similar expressions will be obtained for the two-point function of the chargeconjugate field operators:

$$
\begin{aligned}
& \left\langle 0\left|\phi_{c}(x) \phi_{c}^{*}\left(x^{\prime}\right)\right| 0\right\rangle= \\
& =\sum_{k k^{\prime}}\left\langle 0\left|b_{k} b_{k^{\prime}}^{*} e^{-i k x+i k^{\prime} x^{\prime}}+\left[\alpha_{k} \alpha_{k^{\prime}}^{*}-\delta^{3}\left(\mathbf{k} \mathbf{k}^{\prime}-\mathbf{k}\right)\right] e^{i k x-i k^{\prime} x^{\prime}}\right| 0\right\rangle= \\
& =\sum_{k k^{\prime}}\left\langle 0\left|b_{k} b_{k^{\prime}}^{*} e^{-i k x+i k^{\prime} x^{\prime}}-\delta^{3}\left(\mathbf{k}^{\prime}-\mathbf{k}\right) e^{i k x-i k^{\prime} x^{\prime}}\right| 0\right\rangle= \\
& =-i \tilde{G}\left(x^{\prime}-x\right)=i G\left(x-x^{\prime}\right) .
\end{aligned}
$$

Thus, as a propagator of the complex scalar field formally can be accepted the Pauli-Jordan invariant function $G\left(x^{\prime}-x\right)$ which automatically leads to the chronological ordering and disappears for spacelike intervals:

$$
G\left(x^{\prime}-x\right)=-\tilde{G}\left(x-x^{\prime}\right)=0, \quad\left(x^{\prime}-x\right)^{2}<0 .
$$


The first physical property of a relativistic system which was expressed earlier by the causal propagator $G_{c}\left(x^{\prime}-x\right)$, is that the positive-energy particles should propagate only forward in time, while the negative-energy ones should propagate only backward in time. In our case the propagator $G\left(x^{\prime}-x\right)$ turns to a particle propagator if the time interval lies in the upper light-cone (i.e. $t^{\prime}-t>0$ ) under the moment $t$, and in an antiparticle propagator in opposite case $\left(t^{\prime}-t<0\right)$. In both cases the propagator is retarding with respect to an earlier moment and in this sense the causality requirements are obeyed. Really, in case of an antiparticle the event $t^{\prime}$ happens earlier, than event $t$ and it corresponds to it the retarding propagator with the positive interval $t-t^{\prime}>0$.

The second physical property which the causal propagator should express is that the charge conservation leads to continuity of a world line of a charged particle irrespective of a time direction. In our case the propagator $G\left(x^{\prime}-x\right)$ increases a charge by unity in a world point $x$ and reduces by unity in a world point $x^{\prime}$ whereas $\tilde{G}\left(x^{\prime}-x\right)$ does the inverse. The choice of one of two charge-conjugate propagators $G\left(x^{\prime}-x\right)$ or $\tilde{G}\left(x^{\prime}-x\right)$ depends on to what of two kinds of quanta we assign a positive charge - a particle or an antiparticle. If to a particle it is necessary to use only a propagator $G\left(x^{\prime}-x\right)$ which transfers a positive charge at $t^{\prime}-t>0$, and a negative charge at $t-t^{\prime}>0$.

Thus, the propagator $G\left(x^{\prime}-x\right)$ really can be accepted as the propagator for particles and antiparticles, possessing required causal properties. In the invariant form it has the standard form:

$$
\begin{aligned}
& G\left(x^{\prime}-x\right)=i\left\langle 0\left|\phi\left(x^{\prime}\right) \phi^{*}(x)\right| 0\right\rangle=\frac{i}{(2 \pi)^{3}} \int d^{4} k \varepsilon\left(k_{0}\right) \delta\left(k^{2}-m^{2}\right) e^{-i k x}= \\
& =G_{r e t}\left(x^{\prime}-x\right)-G_{a d v}\left(x^{\prime}-x\right) \text {, } \\
& G_{\text {ret }}\left(x^{\prime}-x\right)=i \int \frac{d^{4} k}{(2 \pi)^{4}} \frac{e^{-i k x}}{m^{2}-k^{2}-i \varepsilon k_{0}} \text {, } \\
& G_{a d v}\left(x^{\prime}-x\right)=i \int \frac{d^{4} k}{(2 \pi)^{4}} \frac{e^{-i k x}}{m^{2}-k^{2}+i \varepsilon k_{0}} \text {. }
\end{aligned}
$$

If now a propagator $G\left(x^{\prime}-x\right)$ to compare to a standard causal propagator $G_{c}\left(x^{\prime}-x\right)$ we see that the causal propagator has one formal and two physically essential unavailability. At first, the chronological ordering in it was made "manually" by inserting of $\theta$ - functions. Secondly, the causal propagator does not disappear for spacelike trajectories and, thirdly, it possesses the strong singularities on small distances $x^{\prime} \rightarrow x$. In the propagator $G\left(x^{\prime}-x\right)$ there are no these deficiencies - the 
chronological ordering happens automatically and uniquely, it disappears behind a light cone and has a weaker behavior on small distances.

\section{Conclusion}

Thus, the $C$-symmetry restrictions lead to new and nontrivial effect - to the natural normal ordering of the creation-annihilation operators of observables of the complex scalar fields with vanishing of the zero-point energy and the zero-point charge of their vacuum. At including of $C$-symmetric interactions the vacuum energy also remains equal to zero.

As a propagator of the complex relativistic field formally can be chosen not a causal propagator, as it has been accepted, but the retired and advanced propagators, having more attractive properties from the physical point of view.

The application of the new method of quantization to other relativistic fields will be considered in forthcoming papers.

\section{References}

1. Zakir Z. (2006) Theor. Phys., Astrophys. and Cosmol., 1, 1, 11; 1, 4, 61; doi: arxiv:0705-0899.

2. Zakir Z. (2007) Theor. Phys., Astrophys. and Cosmol., 2, 2, 9; doi:

3. Zakir Z. (2006) Theor. Phys., Astrophys. and Cosmol., 1, 1, 1; doi: 